\title{
PRIMEROS RESULTADOS DE UN ENSAYO DE SEVERIDAD DE PODA EN Eucalyptus regnans. EFECTOS EN EL CRECIMIENTO Y RESPUESTA FISIOLÓGICA
}

\author{
Lisboa, Magdalena ${ }^{7}$; Acuña, Eduardo ${ }^{1}$; Cancino, Jorge ${ }^{1}$; Muñoz, \\ Fernando ${ }^{1}$; Rodriguez, Roque ${ }^{8}$ y Volker, Peter $^{9}$
}

\section{RESUMEN}

Las crecientes preocupaciones de los consumidores en materias ambientales han llevado a la protección de los bosques naturales. Como resultado, los productores de maderas de alto valor han dirigido su interés a las plantaciones de eucaliptos como una fuente para el suministro de madera de latifoliadas que tradicionalmente se obtenía de bosques naturales.

Varias especies del género Eucalyptus han sido establecidas en diversos países, desde los trópicos hasta las zonas de clima templado frío. Una especie de interés en la zona fría y templada es Eucalyptus regnans, que se ha establecido con éxito en Nueva Zelanda y Chile. En su medio natural, en los bosques húmedos del sureste de Australia, puede alcanzar alturas cercanas a 100 metros y es una de las maderas más populares para una amplia gama de productos, incluidos los pisos, molduras y mobiliario.

En Chile, la producción de madera sólida se basa casi exclusivamente en plantaciones de Pinus radiata, con un pequeño porcentaje de especies nativas y algunas especies del género Eucalyptus. Actualmente existen en la zona central de Chile aproximadamente 1.200 hectáreas de plantaciones de $E$. regnans. La mayor parte de esta superficie se maneja para la producción de madera de alto valor con podas severas e intensivos regímenes de raleo. La poda es una actividad esencial para producir trozas sin defectos que permite la producción de madera y chapas de alto valor.

En las plantaciones de E. regnans destinadas a la producción de madera de alto valor es necesario conocer los efectos de la poda de ramas basales, tanto en las variables de crecimiento como fisiológicas, con el fin de generar madera libre de defectos, sin afectar la productividad de estas plantaciones. En varias especies de Eucalyptus se ha observado que la poda de ramas basales verdes aumenta la tasa fotosintética, entre otros cambios en procesos fisiológicos, lo que podría mitigar el efecto de la pérdida de follaje en el crecimiento.

Para estudiar este efecto, se estableció un ensayo de severidad de poda en rodales de 2 y 3 años de edad, con tratamientos de poda de $0 \%$ (control), 30\%, 50\% y $70 \%$ de remoción de copa viva. Se efectuaron mediciones de diámetro (DAP) y altura al momento de la poda y luego cada tres meses. Adicionalmente, se midió las variables fisiológicas; fotosíntesis, conductancia estomática y transpiración, analizando el efecto del tratamiento, edad del follaje y sección de la copa. Los primeros resultados indican diferencias en las tasas de crecimiento en diámetro entre tratamientos. Los parámetros fisiológicos analizados presentaron diferencias entre edades de follaje, secciones de copa y tratamiento de severidad de poda.

Palabras clave: Euycalyptus regnans, podas, madera sólida.

\footnotetext{
${ }^{7}$ Facultad de Ciencias Forestales, Universidad de Concepción, Concepción, Chile, ${ }^{\star}$ mlisboau@udec.cl

${ }^{8}$ Escuela Politécnica Superior de Lugo. Universidad de Santiago de Compostela 27002, Lugo, España

${ }^{9}$ Cooperative Research Centre for Sustainable Production Forestry and School of Plant Science, University of

Tasmania, GPO Box 252-55, Hobart, 7001, Tas., Australia.
} 


\section{SUMMARY}

Increasing consumer concerns about environmental matters have driven to an also increasing native forests protection. As a result, high value solid wood producers are changing their attention to planted forests, within them Eucalypts plantations, as a broadleave wood source traditionally provided by native forests.

Several Eucalytus species have been established in different countries from the tropics to the temperate zones. One of the interesting Eucalyptus species in temperate areas plantations is Eucalyptus regnans, succesfully planted in countries like New Zealand and Chile. In its natural distribution área in Australian south eastern humid forests, Eucalyptus regnans can reach up to 100 $\mathrm{m}$ height and is one of the most valuable wood to a wide variety of products, including floors, mouldings and furnitures.

Almost all the solid wood production in Chile is based on Radiata Pine plantations with a low native and other exotic species participation including some Eucalypts. At present there are some 1,200 ha of Eucalyptus regnans plantations in the Chile's central part and most of them are used to high value wood production under intensive pruning and thining regimes. Pruning pratice is essential to obtain defect free logs for high value sawn wood and venners production.

In Eucalyptus regnans planted forests to high value wood production it is important the knowledge on the the prunning effect on the growing and phisioplogycal variables in order to produce high value Wood without effects on the plantation productivity. Several Eucalyptus species have shown that basal green branches pruning increases photosintetic rates and that could mitigate the effect of foliage loss on growth.

To study that effect, a pruning severity trial on 2 and 3 years Eucalyptus regnans stands was established, including without pruning as control and 30,50 and $70 \%$ of crown removal treatments. Diameter $(\mathrm{DBH})$ and heigh measures were carried out at the beginnng and later each 3 month. Physiological variables, as photosynthesis, stomatal conductance and transpiration, were measured as well to analyze the treament effects.

First results show that there are differences on growing rates between treatments and regarding to physiological variables, those show differences between foliage age, crown section and pruning severity treatment

Key words: Eucalyptus regnans, pruning, solid wood. 


\section{INTRODUCCIÓN}

La obtención de productos madereros de alto valor requiere maximizar la producción de madera libre de nudos y otros defectos. Debido a que muchas especies del género Eucalyptus no pierden las ramas basales naturalmente, o lo hacen lentamente, la producción de madera libre de defectos a partir de ellas requiere la ejecución de podas. Sin embargo, esta actividad podría facilitar el ingreso de hongos, reduciendo la calidad de la madera por presencia de manchas, y también podría afectar la tasa de crecimiento de los árboles.

La poda de las ramas basales vivas permite controlar el tamaño del cilindro defectuoso y prevenir la formación de nudos sueltos. Podas severas realizadas a edades tempranas permiten minimizar el tamaño del cilindro defectuoso, pero implican una reducción importante del área foliar, variable relacionada con el crecimiento, ya que determina la capacidad del árbol de asimilar carbono, elemento básico para realizar fotosíntesis y producir carbohidratos (Pinkard and Beadle, 1998).

Los árboles tienen la capacidad de disminuir el impacto que produce la poda en el crecimiento, a través de respuestas fisiológicas que incrementan la producción de biomasa (Pinkard and Beadle, 2000). La respuesta fisiológica que ocurre luego de la poda, produce un incremento en la producción de biomasa, debido a cambios en las propiedades de las hojas, en la estructura de copa y, principalmente, en la capacidad fotosintética. Este tipo de respuesta ha sido reportado por diferentes autores. Pinkard et al. (1998) encontraron que Eucalyptus nitens aumentaba hasta en $170 \%$ su tasa fotosintética luego de la poda. Incrementos en la capacidad fotosintética también fueron encontrados en Acacia melanoxylon luego de una poda que redujo la copa en $50 \%$ (Medhurst et al., 2006). Otra variable que se ha medido en respuesta a la poda es la conductancia estomática que generalmente presenta relaciones lineales, logarítmica o potencial con la capacidad fotosintética ((Medhurst et al., 2006; Medrano et al., 2002; Pinkard, 2003)

En Chile existen actualmente más de 1.200 ha de Eucalyptus regnans, superficie que en su mayoría está destinada a productos de madera de alto valor que requieren la ejecución de podas tempranas y severas en las plantaciones. Aunque esa actividad es habitual en plantaciones de esta especie, en el país se desconoce el efecto de la poda sobre la fisiología de los árboles. El objetivo del presente estudio es evaluar el efecto de diferentes severidades de poda en la tasa de asimilación de $\mathrm{CO}_{2}$ y en el crecimiento en diámetro y altura, en plantaciones de Eucalyptus regnans de dos y tres años de edad, destinadas a la producción de madera libre de nudos, ubicadas en la costa de la provincia de Arauco, región del Bío Bío, Chile.

\section{MATERIAL Y MÉTODO}

\section{Descripción del Área de Estudio}

Se instalaron dos ensayos de severidad de poda en plantaciones de E. regnans de dos y tres años de edad, plantados los años 2009 y 2008, respectivamente. Ambos rodales fueron establecidos en el mes de octubre, con distanciamiento de $2,5 \mathrm{~m}$ sobre hilera y con $4 \mathrm{~m}$ y $6 \mathrm{~m}$ entre hileras, para el rodal de dos y tres años, respectivamente. En ambos casos, previo a la plantación se realizó un control de malezas mecánico y se fertilizó al momento del establecimiento. Las plantas de los ensayos tienen la misma procedencia.

Ambos ensayos se encuentran ubicados en la costa de la provincia de Arauco, Región del Bío Bío, Chile (Figura $N^{\circ} 1$ ). El clima de la zona corresponde a templado lluvioso, con influencia mediterránea; la precipitación media anual es $1.330 \mathrm{~mm}$, con un periodo seco de 3 a 4 meses, temperatura media anual de $13^{\circ} \mathrm{C}$, con máximas de $23^{\circ} \mathrm{C}$ en enero y mínimas de $6^{\circ} \mathrm{C}$ en julio. El suelo del sector es del orden Alfisol, originado de terrazas marinas (serie Curanipe). Son suelos con textura franco-arcillosa y arcillosa en profundidad, estructura de bloques subangulares, muy plásticos y adhesivos en húmedo. La topografía es ondulada a quebrada. 


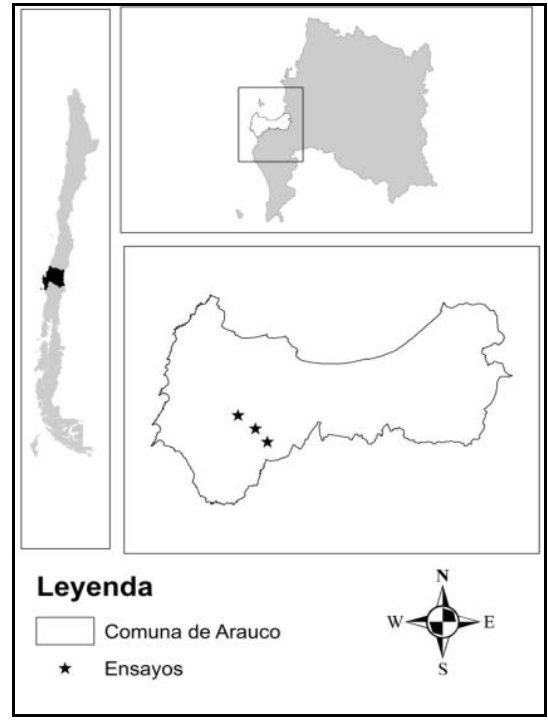

Izquierda: Chile continental

Arriba derecha: Región del Bío Bío

Abajo derecha: Comuna de Arauco

Figura $\mathrm{N}^{\circ} 1$

UBICACIÓN DE LOS ENSAYOS

\section{Diseño Experimental}

En el rodal plantado en 2008 se estableció un ensayo con un diseño en bloques aleatorios completos, con cuatro tratamientos y tres repeticiones; la unidad experimental corresponde a una parcela de 81 árboles (9 hileras de 9 árboles), dos hileras de borde y 25 árboles en el núcleo o parcela de medición (Figura $N^{\circ} 2$ ). En el rodal plantado en 2009, debido a su superficie pequeña, el ensayo se estableció con un diseño completamente al azar, con 15 árboles por tratamiento; la unidad experimental corresponde al árbol.

Cada ensayo incluye cuatro tratamientos de poda, consistentes en la remoción de 0,30 , 50 y $70 \%$ de la copa viva. La poda fue realizada entre los meses de septiembre y octubre de 2011. Detalles de las características de cada rodal y del los tratamientos de poda en cada ensayo son entregados en los Cuadros $\mathrm{N}^{\circ} 1$ y $\mathrm{N}^{\circ} 2$.



Gris claro: Árboles zona buffer

Gris oscuro: 25 árboles parcela de medición.

Figura $\mathrm{N}^{\circ} 2$

UBICACIÓN DE LOS ÁRBOLES EN PARCELA DEL ENSAYO ESTABLECIDO EN EL RODAL DE TRES AÑOS 


\section{Toma y Análisis de Datos}

En cada ensayo se midió la tasa de asimilación de $\mathrm{CO}_{2}$ o tasa fotosintética $(A)$ en tres árboles de área basal cercana al promedio por cada tratamiento de poda.

Las mediciones se realizaron a lo largo de la copa, la cual se dividió longitudinalmente en cuatro secciones de 0 a $30 \%, 30$ a $50 \%, 50-70 \%$ y 70 a $100 \%$.

En el rodal plantado en 2008 las mediciones se realizaron a las 5, 12, 19 y 28 semanas después de la poda.

En el rodal plantado en 2009 las mediciones se realizaron previo a la poda y a las 6, 10, 14 y 18 semanas después de podado.

Además se midió el diámetro a la altura del pecho (DAP) y la altura total $(H)$, estas variables fueron medidas al momento de la poda y luego cada 3 meses en 3 oportunidades, con lo cual se obtuvo el incremento medio mensual tanto en DAP como en altura.

Las mediciones de intercambio gaseoso foliar se realizaron utilizando un IRGA (Infra Red Gas Analyzer) CIRAS-1 (PP Systems, Haverhill, MA). De cada sección de la copa se escogió una rama orientada hacia el norte, en la cual se midieron hojas de tres edades diferentes:

Follaje antiguo, correspondiente a follaje caracterizado por un color verde oscuro, presentando a veces amarillamiento, peciolo marrón y engrosado y el haz muy bien diferenciado del envés.

Follaje maduro, de color verde oscuro, totalmente expandido, con la cutícula bien desarrollada y peciolos de color rojizo

Follaje reciente, correspondiente a hojas de color verde claro, en proceso de expansión, con la cutícula en desarrollo y sin diferenciación entre el haz y el envés.

En los ensayos, no siempre fue posible encontrar las tres edades de follaje en cada sección de copa. En el rodal de dos años, en las 2 primeras mediciones, solo se midió follaje maduro y follaje reciente, los únicos presentes en los árboles; a partir de la medición de la semana 14 se comenzó a medir follaje antiguo.

En las mediciones se utilizó una concentración de $\mathrm{CO}_{2}$ de $380 \mathrm{ppm}$, similar a la concentración que se observa naturalmente en la atmósfera, un flujo de $180 \mu \mathrm{mol} / \mathrm{m}^{2} / \mathrm{s}$ y una densidad de flujo de fotones de $1500 \mu \mathrm{mol} / \mathrm{m}^{2} / \mathrm{s}$ que se obtuvo mediante una fuente de luz externa.

Los datos fueron sometidos a análisis de varianza (ANOVA) y test de separación de medias, Tukey específicamente, para evaluar el efecto de la poda en el crecimiento y en la tasa de asimilación de $\mathrm{CO}_{2}$. En el análisis se utilizó los procedimientos MIXED y GLM del software estadístico SAS. Cada ensayo fue analizado por separado y en ambos casos se analizó como un diseño anidado, donde la sección de copa está anidada dentro del tratamiento y la edad de follaje dentro de la sección de copa.

En el ensayo del rodal de tres años de edad, la variación debida a la hora de medición se incluyó dentro de los bloques, dividiendo el horario de medición en tres intervalos de tiempo (1011:30; 11:30-12.30; 12:30-14 h, en primavera, y 9:30-10:30; 10:30-11:30; 11:30-12:30 h, en verano) y midiendo todos los tratamientos de cada bloque en el mismo periodo de tiempo (modelo 1). El porqué de la selección de este rango de horarios se explica más adelante. En el análisis del ensayo del rodal de dos años se utilizó como co-variable la hora del día en que se tomó la medición (modelo 2). 


$$
\begin{gathered}
A_{i j k l m}=\mu+B_{i}+T_{j}+S_{k(j)}+E_{l(k(j))}+e_{i j k l m} \\
A_{j k l}=\mu+X+T_{j}+S_{k(j)}+E_{l(k(j))}+e_{j k l m}
\end{gathered}
$$

En el modelo [1], válido para el rodal de tres años de edad, $A_{\mathrm{ijklm}}$ es la tasa de asimilación de $\mathrm{CO}_{2}$ de una hoja de un árbol en el bloque $\mathrm{i}$, con tratamiento de poda j, en la sección de copa $\mathrm{k}$ y de edad I; $\mu$ es la media general.

En el modelo [2], válido para el rodal de dos años de edad, $A_{\mathrm{ijkl}}$ es la tasa de asimilación de $\mathrm{CO}_{2}$ de una hoja de un árbol, con tratamiento de poda j, en la sección de copa $\mathrm{k}$ y de edad I.

Cuadro $\mathrm{N}^{\circ} 1$

VARIABLES DE ESTADO DE CADA RODAL AL MOMENTO DE LA PODA

\begin{tabular}{|c|c|c|c|c|c|c|}
\hline Año Plantación & $\begin{array}{c}\text { Edad } \\
\text { (años) }\end{array}$ & $\mathbf{N}$ & $\begin{array}{c}\text { DAP } \\
(\mathbf{c m})\end{array}$ & $\begin{array}{c}\text { DMSM } \\
(\mathbf{c m})\end{array}$ & $\begin{array}{c}\text { H } \\
(\mathbf{m})\end{array}$ & $\begin{array}{c}\text { Hcv } \\
(\mathbf{m})\end{array}$ \\
\hline 2008 & 3 & 660 & $8,9(2,4)$ & $13,3(2,2)$ & $8,4(1,4)$ & $0,20(0,17)$ \\
2009 & 2 & 1000 & $8,0(1,0)$ & $12,5(1,9)$ & $6,5(0,6)$ & $0,09(0,08)$ \\
\hline
\end{tabular}

$\mathrm{N}: \quad$ Número de árboles por hectárea.

DAP: Diámetro a la altura del pecho.

DMSM: Diámetro máximo sobre muñón.

$\mathrm{H}: \quad$ Altura total.

Hcv: $\quad$ Altura de inicio de la copa viva.

(): $\quad$ Desviación estándar de cada variable.

Cuadro $\mathrm{N}^{\circ} 2$

\begin{tabular}{|c|c|c|c|c|c|c|}
\hline Tratamiento & \multicolumn{2}{|c|}{ T1 } & \multicolumn{2}{|c|}{ T2 } & \multicolumn{2}{|c|}{ T3 } \\
\hline Año de Plantación & $\begin{array}{c}\begin{array}{c}\text { Altura } \\
\text { de Poda }\end{array} \\
\text { (m) }\end{array}$ & 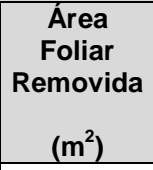 & $\begin{array}{c}\begin{array}{c}\text { Altura } \\
\text { de } \\
\text { Poda }\end{array} \\
\text { (m) } \\
\end{array}$ & 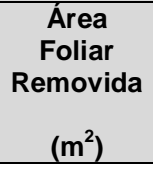 & $\begin{array}{c}\begin{array}{c}\text { Altura } \\
\text { de } \\
\text { Poda }\end{array} \\
\text { (m) } \\
\end{array}$ & 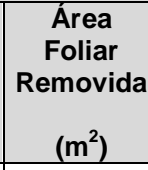 \\
\hline 2008 & 2,6 & 10,70 & 4,7 & 19,84 & 6,4 & 25,68 \\
\hline 2009 & 2,0 & 9,55 & 3,1 & 12,35 & 4,6 & 14,84 \\
\hline Copa Viva Removida (\%) & \multicolumn{2}{|c|}{30} & \multicolumn{2}{|c|}{50} & \multicolumn{2}{|c|}{70} \\
\hline
\end{tabular}

ALTURA PROMEDIO DE PODA Y ÁREA FOLIAR REMOVIDA SEGÚN TRATAMIENTO EN CADA ENSAYO 


\section{RESULTADOS Y DISCUSIÓN}

\section{Efecto de la Severidad de Poda en el Incremento Medio Mensual en Diámetro Altura Total}

En ambos rodales se observa una disminución del incremento en DAP al aumentar la severidad de poda, variando desde $0,1 \mathrm{~cm} /$ mes a $3,5 \mathrm{~cm} / \mathrm{mes}$ entre el tratamiento de $70 \%$ de remoción de copa y el testigo para el rodal establecido el año 2008 y desde 0,15 a $0,37 \mathrm{~cm} / \mathrm{mes}$ para el rodal establecido el año 2009.

En el rodal establecido el año 2008 al remover un 30\% de la copa se produce un aumento del incremento en DAP en comparación al testigo, lo cual se puede deber a que al remover esta porción de la copa se está eliminando principalmente follaje antiguo que no aporta a la productividad de la copa (Figura $\mathrm{N}^{\circ} 3 \mathrm{a}$ ).

Para el incremento en altura, sólo se observa una tendencia clara en el rodal establecido el año 2008, para el cual a mayor severidad de poda hay un menor incremento mensual en altura, variando desde 0,39 a $0,25 \mathrm{~m} / \mathrm{mes}$ entre el testigo y el tratamiento de mayor severidad de poda (Figura $\mathrm{N}^{\circ} 3 \mathrm{~b}$ ).

Pinkard et al. (2004) encontraron que para E. nitens no se ve afectado su crecimiento tanto en DAP como en altura al remover el $50 \%$ de su copa, en cambio para E. globulus es recomendable podar solo el $30 \%$ de la copa.


Figura $\mathbf{N}^{\circ} 3$

RELACIÓN ENTRE EL PORCENTAJE DE REMOCIÓN DE COPA

Y EL INCREMENTO MEDIO MENSUAL EN DAP Y ALTURA TOTAL

\section{Efecto de la Severidad de Poda en las Tasas de Asimilación de $\mathrm{CO}_{2}$}

En general, independientemente de la edad del rodal, se encontraron diferencias significativas $(p<0,05)$ en las tasas de asimilación de $\mathrm{CO}_{2}$ entre las diferentes edades de follaje (Cuadro $N^{\circ} 3$ y Cuadro $N^{\circ} 4$ ). 
Cuadro $\mathrm{N}^{\circ} 3$

ANÁLISIS DE VARIANZA PARA EL EFECTO DEL TRATAMIENTO DE PODA, EDAD DE FOLLAJE Y SECCIÓN DE COPA EN LA TASA DE ASIMILACIÓN DE $\mathrm{CO}_{2}$

LUEGO DE 5, 12, 19 Y 28 SEMANAS DE REALIZADA LA PODA ENSAYO REALIZADO EN LA PLANTACIÓN DE TRES AÑOS DE EDAD

\begin{tabular}{|l|c|c|c|c|}
\hline Semana (Día) & $\mathbf{5 ( 3 5 )}$ & $\mathbf{1 2 ( 8 4 )}$ & $\mathbf{1 9}(\mathbf{1 3 9})$ & $\mathbf{2 8}(\mathbf{2 0 2})$ \\
\hline Efecto & $\operatorname{Pr}(\mathbf{F})$ & $\operatorname{Pr}(\mathbf{F})$ & $\operatorname{Pr}(\mathbf{F})$ & $\operatorname{Pr}>\mathbf{F}$ \\
\hline Bloque & $<0,0001(16,76)$ & $0,0003(8,54)$ & $0,0023(6,41)$ & $0,0001(10,51)$ \\
Tratamiento & $0,0726(2,37)$ & $<0,0001(15,16)$ & $<0,0001(17,02)$ & $<0,0001(24,10)$ \\
Sección (tratamiento) & $0,0014(3,83)$ & $0,2792(1,29)$ & $0,0001(5,03)$ & $<0,0001(15,43)$ \\
Edad (TratamientoxSección) & $<0,0001(5,43)$ & $<0,00012,35)$ & $<0,0001(13,88)$ & $<0,0001(17,78)$ \\
\hline
\end{tabular}

\section{Cuadro $\mathrm{N}^{\circ} 4$}

ANÁLISIS DE VARIANZA PARA EL EFECTO DEL TRATAMIENTO DE PODA, EDAD DE FOLLAJE Y SECCIÓN DE COPA EN LA TASA DE ASIMILACIÓN DE $\mathrm{CO}_{2}$ AL MOMENTO DE REALIZAR LA PODA Y LUEGO DE 6, 10, 14 Y 18 SEMANAS DE REALIZADA LA PODA ENSAYO REALIZADO EN LA PLANTACIÓN DE DOS AÑOS DE EDAD

\begin{tabular}{|l|c|c|c|c|c|}
\hline Semana (Día) & $\mathbf{0 ( 0 )}$ & $\mathbf{6 ( 5 3 )}$ & $\mathbf{1 0}(\mathbf{7 8})$ & $\mathbf{1 4}(\mathbf{1 0 9})$ & $\mathbf{1 8}(\mathbf{1 4 2})$ \\
\hline Efecto & $\operatorname{Pr}(\mathbf{F})$ & $\operatorname{Pr}(\mathbf{F})$ & $\operatorname{Pr}(\mathbf{F})$ & $\operatorname{Pr}(\mathbf{F})$ & $\operatorname{Pr}(\mathbf{F})$ \\
\hline Hora & $0,9461(0,00)$ & $0,0040(8,83)$ & $<0,0001(21,98)$ & $0,0002(15,09)$ & $0,5649(0,33)$ \\
Tratamiento & $0,0872(2,29)$ & $<0,0001(16,61)$ & $<0,0001(11,14)$ & $<0,0001(8,66)$ & $<0,0001(8,40)$ \\
Sección (tratamiento) & $0,6288(0,73)$ & $0,6478(0,70)$ & $0,0014(3,85)$ & $0,0077(3,09)$ & $0,2735(1,28)$ \\
Edad (TratamientoxSección) & $<0,0001(35,1)$ & $0,0054(3,63)$ & $<0,0001(10,01)$ & $<0,0001(15,67)$ & $<0,0001(5,55)$ \\
\hline
\end{tabular}

Las hojas maduras presentaron la mayor tasa de asimilación de $\mathrm{CO}_{2}(A)$, seguidas por el follaje reciente y en tercer lugar el follaje antiguo (Figura $\mathrm{N}^{\circ} 4$ ).

Estos resultados son concordantes con los obtenidos en estudios realizados en otras especies. Por ejemplo, Pinkard et al. (1998) en Eucalyptus nitens detectaron que en general el follaje antiguo presentó los menores valores de $A$, seguido por el follaje más reciente y luego por el maduro.

En Acacia melanoxylon también se encontró que el follaje reciente presenta valores de $A$ mayores al follaje antiguo (Medhurst et al., 2006).

Las bajas tasas de $A$ observadas en el follaje antiguo pueden explicarse, en parte, por la localización del mismo; la mayor parte de follaje antiguo se localiza en la parte baja de la copa, sección que recibe menor radiación, lo que determinaría una apertura incompleta de los estomas.

Estas bajas tasas también podrían deberse a una pérdida de la función en el follaje antiguo (England and Attiwill, 2006). 


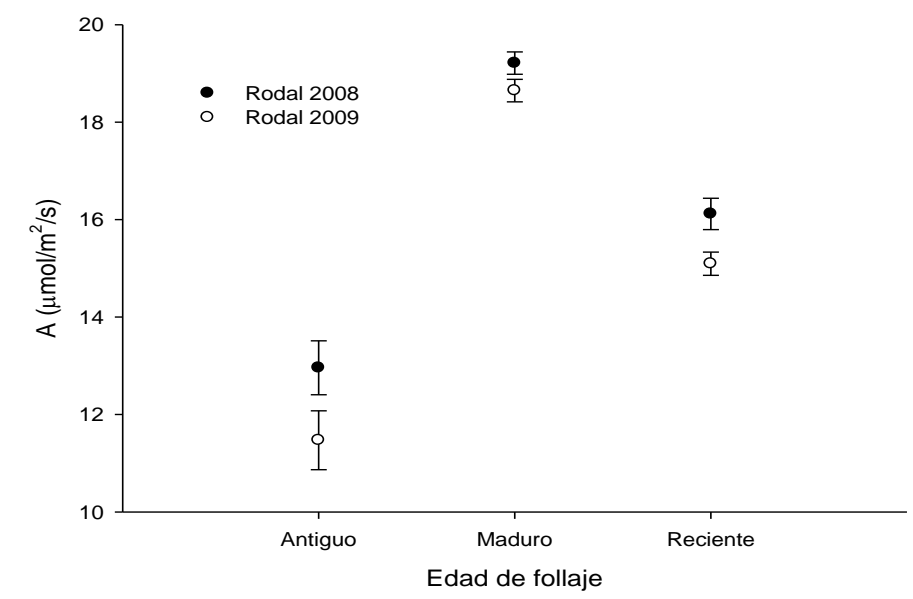

Figura $N^{\circ} 4$

TASA DE ASIMILACIÓN DE $\mathrm{CO}_{2}$ SEGÚN EDAD DE FOLLAJE PARA AMBOS RODALES

En ambos rodales, las variables fisiológicas observadas variaron ampliamente entre secciones de copa. En el rodal de 2 años, la tasa de asimilación de $\mathrm{CO}_{2}$ fue mayor a mayor altura en la copa, en cambio en el rodal de 3 años los mayores valores los presentó la sección de copa 3 $(50-70 \%)$ (Figura N ${ }^{\circ}$ ).

Los bajos valores de $A$ presentados en la sección 1, pueden deberse a la gran cantidad de follaje antiguo presente y a la pequeña cantidad de luz que llega a esta sección de la copa; le sigue la sección 4 que es la más expuesta a la radiación y además es la zona donde se acumula la mayor cantidad de follaje reciente, el que aún no se ha desarrollado por completo y no se encuentra fotosintetizando a su máxima capacidad.

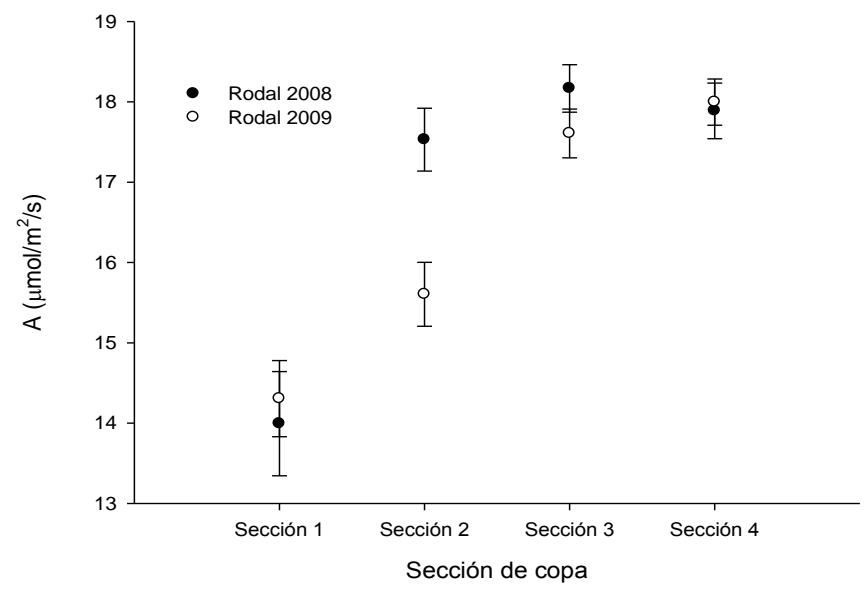

TASA DE ASIMILACIÓN DE $\mathrm{CO}_{2}$ (A) SEGÚN SECCIÓN DE COPA PARA AMBOS RODALES 
En ambos rodales se observa una amplia variación de la tasa de asimilación de $\mathrm{CO}_{2}(A)$ entre tratamiento a lo largo del tiempo. En ambos rodales, los árboles del tratamiento testigo presentaron la menor tasa de asimilación de $\mathrm{CO}_{2}$ en todas las instancias de medición (Figura $\mathrm{N}^{\circ} 6$ y Figura $N^{\circ} 7$ ).

En el rodal de dos años después de realizada la poda, los árboles con 30, 50 y $70 \%$ de remoción de copa aumentaron sus valores de $A$ (Figura $N^{\circ} 7$ ).

Los mayores valores de $A$ para ambos ensayos los presentaron los árboles del tratamiento con remoción de copa de $70 \%$ (T3) con valores hasta $50 \%$ mayores que los del tratamiento testigo, excepto en la última medición del rodal de dos años, donde el tratamiento con remoción de 50\% de la copa, alcanzó los mayores valores (Figura 6 y Figura 7).

En general la magnitud de la respuesta en $A$ a la poda fue mayor en el rodal de tres años.

El efecto del tratamiento de poda en las tasas de asimilación de $\mathrm{CO}_{2}$ en el ensayo de tres años, solo fue significativo $(p<0,05)$ desde la semana 12 en adelante (Cuadro $\left.N^{\circ} 3\right)$.

La alta significancia que muestra el efecto del bloque, afirma la necesidad de dividir en rangos de tiempo las mediciones, debido a la amplia variación que hay en las tasas fotosintéticas a lo largo del día.

En el rodal de dos años, la poda produjo un efecto significativo en la tasa fotosintética ( $p$ $<0,05$ ) desde la segunda medición en adelante (Cuadro $\mathrm{N}^{\circ} 4$ ).

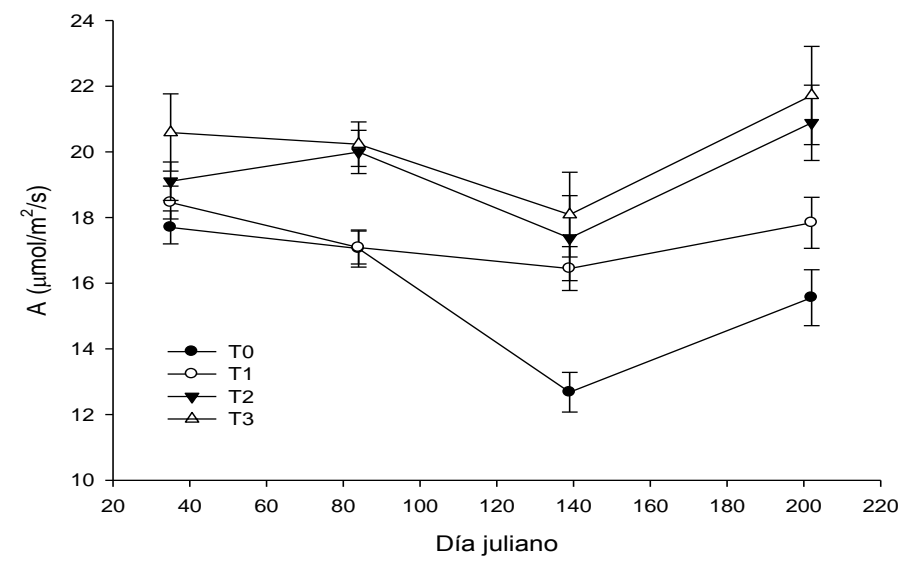

Figura $\mathrm{N}^{\circ} 6$

VARIABLES FISIOLÓGICAS EN EL ENSAYO REALIZADO EN LA PLANTACIÓN DE TRES AÑOS DE EDAD 5, 12, 19 Y 28 SEMANAS DESPUÉS DE REALIZADA LA PODA 


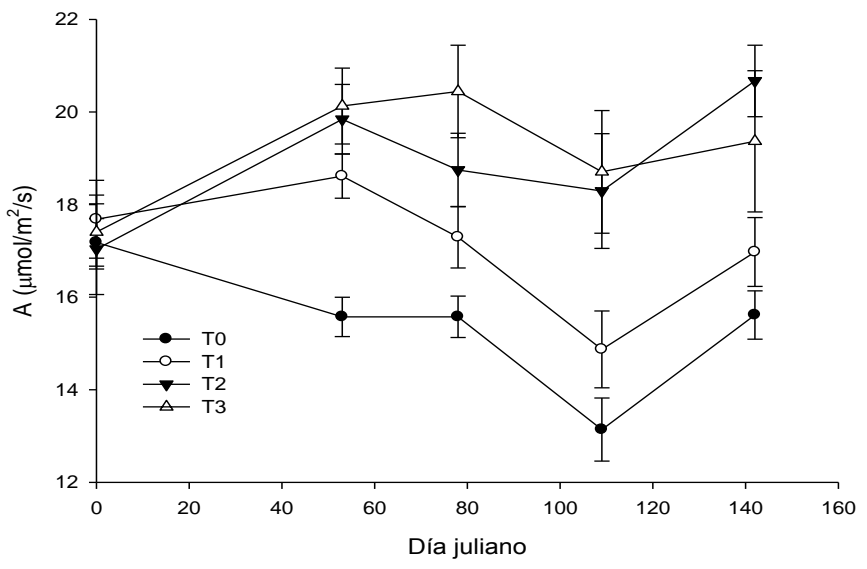

Figura $\mathrm{N}^{\circ} 7$

VARIABLES FISIOLÓGICAS DEL ENSAYO REALIZADO EN LA PLANTACIÓN DE DOS AÑOS DE EDAD EN EL MOMENTO DE REALIZAR LA PODA Y 6, 10, 14 Y 18 SEMANAS LUEGO DE LA PODA

En Australia se ha estudiado el efecto de la poda en la tasa fotosintética $(A)$ de algunas especies de Eucalyptus, encontrando diversas respuestas. Mientras que Pinkard et al. (1998) encontraron que Eucalyptus nitens aumentaba hasta en $170 \%$ su tasa fotosintética luego de la poda, Forrester et al. (2012) encontraron solo un aumento de $19 \%$ en la tasa fotosintética 19 semanas luego de podar el $50 \%$ de la copa viva de Eucalyptus nitens. Incrementos en la capacidad fotosintética también fueron encontrados en Acacia melanoxylon luego de podar el $50 \%$ de su copa (Medhurst et al., 2006). Contrariamente a lo que sucede en las especies mencionadas, Alcorn et al. (2008) encontraron que la poda del $50 \%$ de la copa viva de Eucalyptus cloeziana y Eucalyptus pilularis, no produce aumentos en las tasas de asimilación de $\mathrm{CO}_{2}$ hasta 13 meses después de realizada la poda.

Pinkard et al. (1998) observaron que árboles de menor edad presentaban respuestas a la poda de mayor magnitud que un rodal de mayor edad, a diferencia de lo encontrado en este estudio, donde el rodal de tres años en general presentó una respuesta de mayor magnitud que el de dos años.

\section{CONCLUSIONES}

La poda de ramas vivas en Eucalyptus regnans aumenta la tasa de asimilación de $\mathrm{CO}_{2}, \mathrm{~A}$ mayor severidad de poda, mayor tasa de asimilación.

La edad del follaje es determinante en las tasas de asimilación de $\mathrm{CO}_{2}$. En orden decreciente es más relevante el follaje maduro, el reciente y por último el antiguo.

La tasa se asimilación varía entre secciones de copa. En general se observa que los mayores valores de tasas de asimilación de $\mathrm{CO}_{2}$ los presenta la sección de copa 3 (70-90\%) y los menores la sección 1 (0-30\%), pero las diferencias entre secciones no fueron significativas en todas las mediciones. 


\section{REFERENCIAS}

Alcorn, P. J.; Bauhus, J.; Thomas, D. S.; James, R. N.; Smith, R. G. B. and Nicotra, A. B., 2008. Photosynthetic response to green crown pruning in young plantation-grown Eucalyptus pilularis and E. cloeziana. Forest Ecology and Management (2008) 255:3827-3838.

England, J. and Attiwill, P., 2006. Changes in leaf morphology and anatomy with tree age and height in the broadleaved evergreen species. Eucalyptus regnans F. Muell. Trees - Structure and Function 20:79-90.

Forrester, D.I.; Collopy, J.J.; Beadle, C.L.; Warren, C.R. and Baker, T. G., 2012. Effect of thinning, pruning and nitrogen fertilizer application on transpiration, photosynthesis and water-use efficiency in a young Eucalyptus nitens plantation. Forest Ecology and Management (2012) 266:286-300.

Medhurst, J.; Pinkard, E.; Beadle, C. and Worledge, D., 2006. Photosynthetic capacity increases in Acacia melanoxylon following form pruning in a two-species plantation. Forest Ecology and Management (2006) 233:250259.

Medrano, H.; Escalona, J.; Bota, J.; Gulías, J.and Flexas, J., 2002. Regulation of Photosynthesis of C3 Plants in Response to Progressive Drought: Stomatal Conductance as a Reference Parameter. Annals of Botany (2002) 89:895-905.

Pinkard, E., 2003. Physiological and growth responses related to pattern and severity of green pruning in young Eucalyptus globulus. Forest Ecology and Management (2003) 182:231-245.

Pinkard, E. A. and Beadle, C. L., 1998. Effects of green pruning on growth and stem shape of Eucalyptus nitens (Deane and Maiden) Maiden. New Forests (1998) 15:107-126.

Pinkard, E. and Beadle, C. A., 2000. Physiological approach to pruning. International Forestry Review (2000) 2:295-303.

Pinkard, E.; Beadle, C.; Davidson, N. and Battaglia, M., 1998. Photosynthetic responses of Eucalyptus nitens (Deane and Maiden) Maiden to green pruning. Trees-Structure and Function (1998) 12:119-129.

Pinkard, E.; Mohammed, C.; Beadle, C.; Hall, M.; Worledge, D. and Mollon, A., 2004. Growth responses, physiology and decay associated with pruning plantation-grown Eucalyptus globulus Labill. and E. nitens (Deane and Maiden) Maiden. Forest Ecology and Management (2004) 200:263-277. 